\title{
A Fast Method of Aerodynamic Computation for Compound Gyroplane
}

\author{
MA Tielin ${ }^{1,}$ a , HAO Shuai ${ }^{2}$, XUE Pu ${ }^{2}$, LI Gen $^{2}$, GAN Wenbiao ${ }^{1}$ \\ ${ }^{1}$ Research Institute of Unmanned Aerial Vehicle, Beijing University of Aeronautics and Astronautics, \\ Beijing 100191,China \\ ${ }^{2}$ School of Aeronautic Science and Engineering, Beijing University of Aeronautics and Astronautics, \\ Beijing 100191, China \\ aemail:matielin@buaa.edu.cn
}

Keywords: Compound gyroplane; Engineering estimation method; Blade element momentum theory; Fast method; CFD

\begin{abstract}
A fast method of aerodynamic computation (FMAC) is put forward for compound gyroplane. It combines advantages of the blade element momentum theory and the engineering estimation method. To fast obtain aerodynamic characteristics of the compound gyroplane, the FMAC simplifies the interface between rotor and wing, and uses the estimation method to analyze wing and the blade element momentum theory integration method for rotor. Based on the FMAC, aerodynamic characteristics of a certain compound gyroplane are researched, and compares with the sliding mesh CFD method. The comparison confirmed the effectiveness and rapidness of this method. It is shown that the FMAC can be used for rapid aerodynamic analysis and design optimization of compound gyroplane.
\end{abstract}

\section{Introduction}

The compound gyroplane is a combination of gyroplane and fixed-wing aircraft. It has advantages of the both flight vehicles, and uses autorotation rotor and fixed wing as lifting surface. The flight performance of the aircraft is similar with gyroplane at low speed. When it is flying at high speed, collective pitch of rotor is reduced, so the rotor is unloaded to avoid disadvantages such as wingtip stalling. Thus the drag of forward flight is reduced, and the fixed wing undertakes most of the weight[1]. Because the compound rotorcraft has configuration features of both fixed-wing aircraft and rotorcraft, the fast and effective method of aerodynamic analysis becomes a key and difficult point of gyroplane design.

Many experimental and theoretical aerodynamic researches of compound gyroplane have been carried out by foreign researchers. In the course of developing XV-1, McDonnell-Douglas corporation conducted some system researches on the three flight modes of the aircraft, and released a series of aerodynamic performance reports. The corporation also studied the interference of rotor and fuselage[2-4]. $\mathrm{Vu}[5]$ investigated aerodynamic characteristics of a compound gyroplane in preliminary design and optimization phrase. Based on Pitt/Peters dynamic inflow theory numerical integration method, McCormick[6] carried out aerodynamic numerical simulation analysis of autorotation rotor. Kim[7] used a numerical integration method based on non-uniform inflow model to analyze the rotor aerodynamic performance.

Domestic researchers have done many theoretical studies on aerodynamic characteristics of tiltrotor and compound helicopter, whose configuration are similar with compound gyroplane. Based on Weissinger-L lifting surface theory and rolled tip vortex model, Li[8] proposed a free-wake analysis method of tiltrotor and analyzed the steady wake at hover and forward flight status, and the unsteady wake at transition flight status.

Modern CFD method has higher reliability for aerodynamic analysis, but costs lots of computing time, especially for this unconventional configuration vehicle. To calculate unsteady aerodynamic characteristics of rotor, the sliding mesh technology is required, which makes CFD method complex and inefficient. Besides, the variations of rotor pitch and the rotor shaft caster angle increase the computing states, and makes calculating time multiplied. 
However, there isn't fast aerodynamic method of the compound gyroplane now. It is necessary to propose a fast method of aerodynamic computation, which can be used for rapid analysis and provide references for compound gyroplane design and optimization.

\section{FMAC for Compound Gyroplane}

The 3D model of one certain compound gyroplane is shown in Figure 1. The aerodynamic analysis of the rotor and the fixed wing has been carried out. The aerodynamic interference of the components has been discussed. And the fast method is obtained to evaluate the aerodynamic characteristics of the whole aircraft. In this paper, the rotor section only refers to the autorotating rotor part, while the fixed wing section refers to the rest parts, including fixed wing, fuselage and tail.

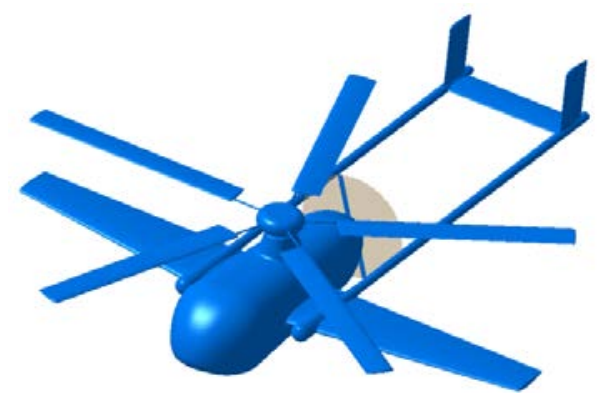

Fig.1 3D Model of the Compound Gyroplane

\section{Theoretical Calculation of Aerodynamic Characteristics of the Rotor Section}

The aerodynamic characteristics of the rotor part can be quickly acquired using the integration method, which is based on blade element momentum theory. The decomposition of aerodynamic force and inlet-air velocity on the blade profile is shown in Figure 2.

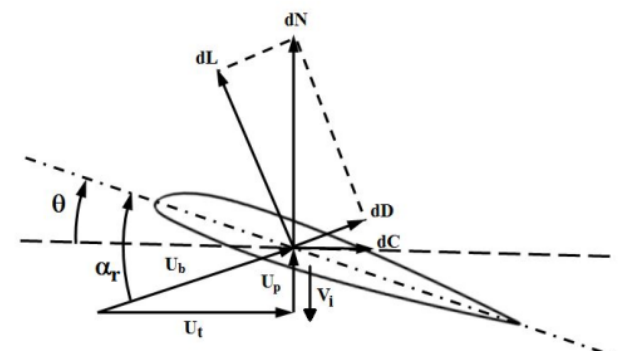

Fig2. Decomposition of Aerodynamic Force and Inlet-air Velocity on the Blade Profile

Where $d \boldsymbol{L}$ is the lift, $\boldsymbol{d D}$ is the drag; $\boldsymbol{d N}$ is the vertical component of the aerodynamic force on the profile, $\boldsymbol{d C}$ is the tangential component; $U_{p}$ is the vertical component of the inlet-air velocity, $U_{t}$ is the tangential component; $U_{b}$ is the resultant inlet-air velocity; $V_{i}$ is the induced velocity; $\boldsymbol{\theta}$ is the collective pitch; $\boldsymbol{\alpha}_{r}$ is the equivalent angle of attack on the profile.

The equation of induced inflow ratio[9] based on blade element momentum theory is:

$$
\lambda_{i}=\sqrt{\left(\frac{\sigma a}{16}-\frac{\lambda_{c l}}{2}\right)^{2}+\frac{\sigma a}{8} \theta r}-\left(\frac{\sigma a}{16}+\frac{\lambda_{c l}}{2}\right)
$$

Where $\lambda_{c l}=\lambda-\lambda_{i}$ is the climb inflow ratio; $\lambda_{i}=\frac{V_{i}}{V_{\text {tip }}}$ is the induced inflow ratio; $\sigma$ is the rotor disk solidity; $\boldsymbol{a}$ is the lift curve slope of the airfoil; $r$ is distance from blade element to the center of propeller shaft.

When the rotor achieved a steady status in the forward flight, the torque of the shaft should be zero, that is:

$$
Q=\frac{K}{2 \pi} \int_{0}^{2 \pi} \int_{0}^{R}[r \cos \beta d C] d \phi d r=0
$$


Where $K$ is the number of blade; $\beta$ is the waving angle.

By solving equation (2), the steady speed of rotor at different forward flight status can be obtained, and the lift $L$ and the drag $D$ of the rotor can be derived as follows:

$$
\begin{gathered}
L=\frac{K}{2 \pi} \int_{0}^{2 \pi} \int_{0}^{R}(d N \cos \beta) d \phi d r \\
D=\frac{K}{2 \pi} \int_{0}^{2 \pi} \int_{0}^{R}(d N \sin \beta \cos \phi-d C \sin \phi) d \phi d r
\end{gathered}
$$

\section{The engineering Estimation method of Fixed Wing Section}

The engineering estimation method is a simple, rapid and effective approach to obtain aerodynamic characteristics [10]. Based on the existing engineering estimation methods [11, 12, 13], the lift coefficient of the fixed wing section can be expressed as:

$$
C_{L}=C_{L, F}^{\alpha} \alpha+C_{L, \mathrm{~W}}^{\alpha}\left(\alpha-\alpha_{0}\right)+C_{L, H}^{\alpha}\left(\left(1-\frac{d \varepsilon}{d \alpha}\right) \alpha+\phi_{H}\right)
$$

Where $C_{L, F}^{\alpha}$ is the slope of lift curve of the fuselage; $C_{L, W}^{\alpha}$ is the slope of lift curve of the wing; $C_{L, H}^{\alpha}$ is the slope of lift curve of the tail; $\alpha$ is the angle of attack of inflow; $\alpha_{0}$ is the zero-lift angle of the wing; $\frac{d \varepsilon}{d \alpha}$ is the variation rate of the wing downwash at the tail; $\phi_{H}$ is the installation angle of the tail.

The drag coefficient of the fixed wing is:

$$
C_{D}=C_{D 0}+C_{D i}
$$

Where $C_{D 0}$ is the zero-lift drag coefficient; $C_{D i}$ is the induced drag coefficient. The induced drag coefficient of the whole section is:

$$
C_{D i}=k^{\prime} C_{L}^{2}+k^{\prime \prime}\left(C_{L}-C_{L \min }\right)^{2}
$$

Where $C_{L \text { min }}$ is the lift coefficient when the drag of the whole section is minimum, generally, $C_{L \min }=C_{L, \alpha=0} ; k^{\prime}$ is the induced drag coefficient; $k^{\prime \prime}$ is the viscous resistance coefficient.

\section{The fast aerodynamic method of Whole Aircraft}

The aerodynamic interference of the rotor and the fixed wing was given priority.

The fuselage is not the main part providing lift, but it can produce large drag-mainly viscous drag. The aerodynamic interference between rotor and fuselage mainly affect lift and pressure drag. So the interference of rotor to fuselage can be ignored, when we are carrying out a rapid calculation of the whole vehicle.

The tail can be affected by the rotor downwash. But the tail of this certain compound gyroplane is located in the propeller wake zone. The propeller wake greatly weakens the influence of rotor downwash at the tail. So the interference between rotor and tail can be ignored.

Therefore, aerodynamic analysis mainly considers the interference of rotor and wing. The wing has the blocking effect on the rotor in flight, and the effect decreases with the forward speed increasing. When the speed is greater than $9.15 \mathrm{~m} / \mathrm{s}$, the interference between wing and the flowfield around rotor can be ignored[14].

The interference between rotor and the fixed wing section is relevant to the rotor downwash influence zone. The influence zone is an oblique backward inclined cylinder (as Fig. 3). The tilt angle of this cylinder is mainly relevant to the forward speed, and the approximate calculation is:

$$
\tan \chi=\frac{V_{0} \sin \delta_{S}-V_{i . t i p}}{V_{0} \cos \delta_{S}}
$$

Where $V_{0}$ is the forward speed; $\delta_{S}$ is the rotor shaft caster angle; $V_{i . t i p}$ is the induced speed of the blade tip. 


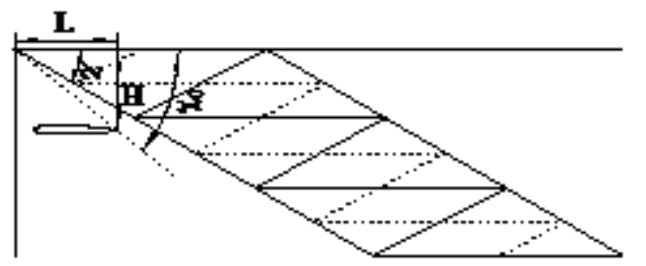

Fig.3 Affected Area of Rotor Downwash

As Fig. 3, $\boldsymbol{H}$ is the horizontal height between wing and rotor; $\boldsymbol{L}$ is the distance from the trailing edge of wing to the leading edge of rotor disk.

According to the compound gyroplane model, the angle between the trailing edge of wing and the front part of rotor disk is:

$$
\chi_{0}=\arctan \left(\frac{H}{L}\right)
$$

By using equation (8) and (9) to calculate the compound gyroplane, we get $\chi=14^{\circ}, \chi_{\mathbf{0}}=26.5^{\circ}$, $\chi_{0}>\chi$. The wing is away from the effected zone of the rotor downwash. So when making fast aerodynamic calculation, the aerodynamic interference between rotor and wing of this certain compound gyroplane can be put away.

In summary, based on this compound gyroplane, aerodynamic characteristics can be acquired rapidly by superimposing forces of the rotor section and the fixed wing section.

\section{The comparison of the results}

Short takeoff and high-speed cruise are important flight sates for compound gyroplane[15], therefore the aerodynamic performances at takeoff and cruise state should be studied.

To verify effectiveness of this method, FMAC and CFD were respectively applied to calculate the aerodynamics at takeoff and cruise state.

Based on SA turbulence model, Reynolds average N-S equation CFD method was chosen to conduct calculation. The flowfield was divided into two parts(rotor area and exterior rotor area). Structured grid and sliding mesh technique was employed to numerical simulation.

\section{The Comparison of Calculation Results at Takeoff State}

The parameters of takeoff are: takeoff height $\mathrm{H}=100 \mathrm{~m}$, takeoff weight $\mathrm{G}=5 \mathrm{t}$, takeoff speed $\mathrm{V}=40 \mathrm{~m} / \mathrm{s}$, rotor shaft caster angle $8^{\circ}$, collective pitch $8^{\circ}$.

The CFD method was used to calculate the whole model with and without rotor section. The streamlines and static pressure distribution of wing middle-section were obtained (as Fig. 4). The streamlines and pressure distribution on the wing surface were shown in Fig. 5. As shown in Fig. 4 and Fig. 5, the streamlines and static pressure distribution have no significant changes with and without rotor.

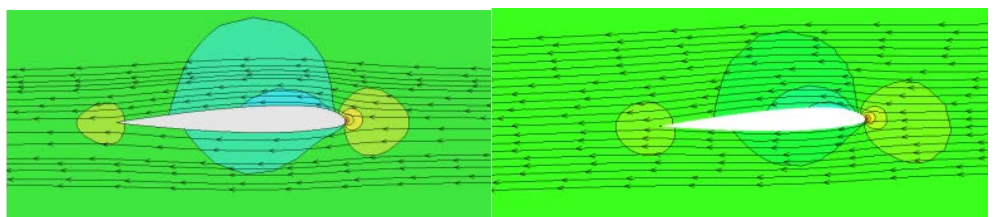

Figure.4 Comparison of Static Pressure Distribution and Streamlines of Wing Middle Section
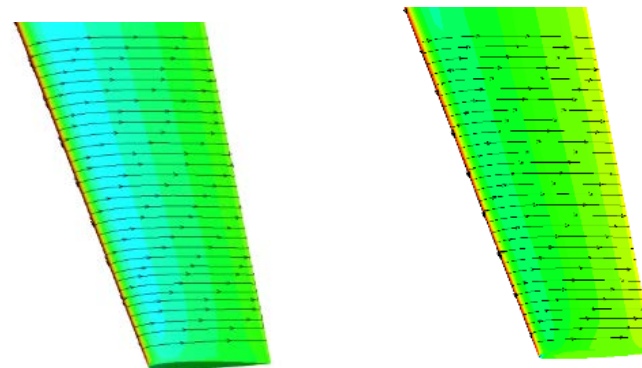

Figure.5 Comparison of Static Pressure Distribution and Streamlines on Wing Surface 
Obviously, there isn't obvious interference caused by rotor downwash, which suggests that the assumption of FMAC is reasonable.

FMAC and CFD method were respectively used to calculate the aerodynamics of whole aircraft at rotor shaft caster angle $8^{\circ}$ and collective pitch $2 \sim 8^{\circ}$ (as Fig. 6).

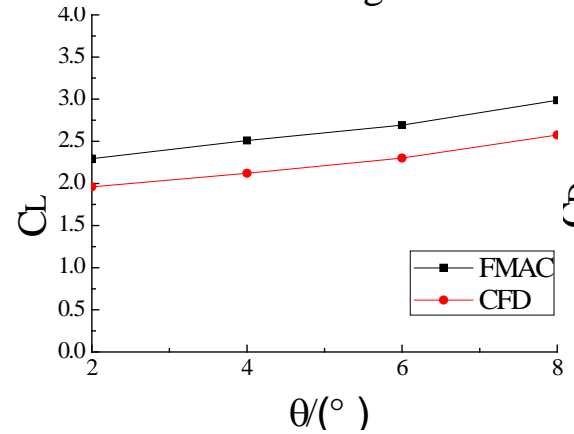

(a) Lift Coefficient

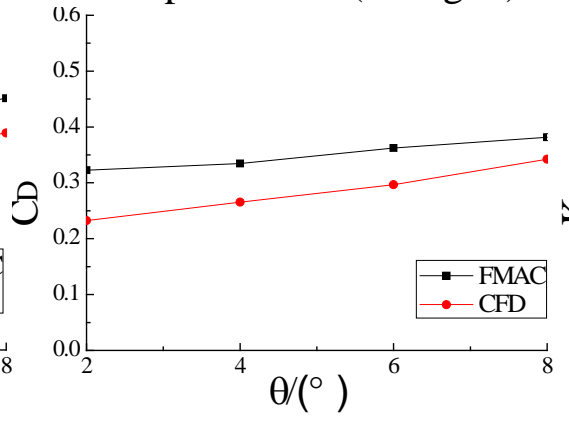

(b) Drag Coefficient

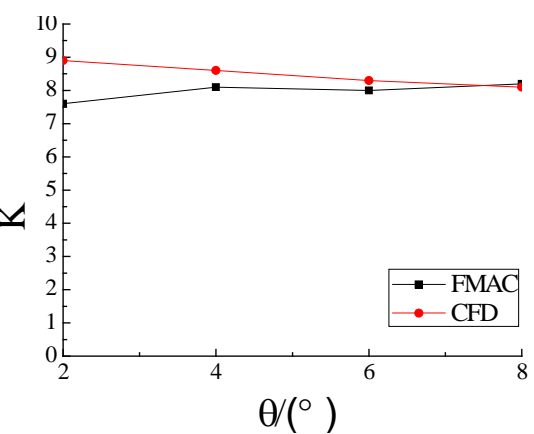

(c) Lift-drag ratio

Figure.6 The Comparison of Aerodynamic Characteristics at Takeoff Status

As shown in Fig. 6, lift and drag characteristics by FMAC and CFD maintain the same trend. The lift and drag of FMAC are about 15\% larger than the CFD results. In the primarily optimization design phrase, this error can be acceptable. But FMAC requires much less computing time than CFD method. It proves that FMAC method is simple, rapid and effective.

\section{The Aerodynamic Characteristics at Cruise State}

FMAC and CFD method are utilized to compute the aerodynamic characteristics at cruise state. The parameters are: cruising altitude $\mathrm{H}=1 \mathrm{~km}$, cruising speed $\mathrm{V}=60 \mathrm{~m} / \mathrm{s}$, cruising weight $\mathrm{G}=4.5 \mathrm{t}$, the collective pitch $2^{\circ}$, the rotor shaft caster angle $2^{\circ}$. The results are shown in figure 7 .

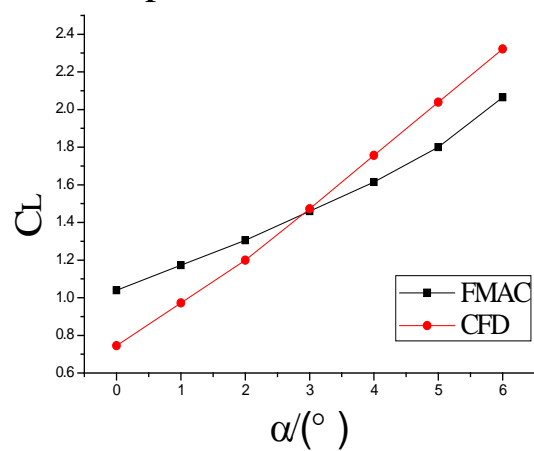

(a) Lift Coefficient

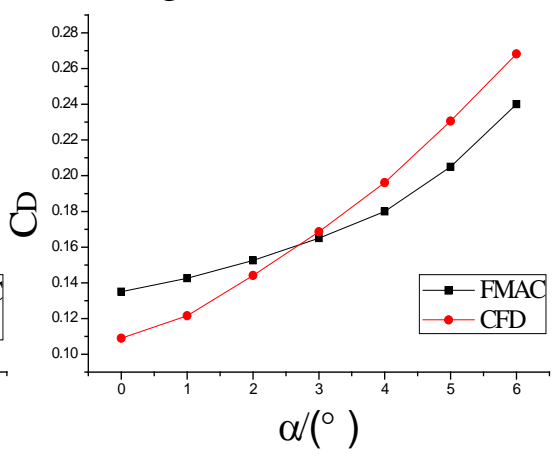

(b) Drag Coefficient

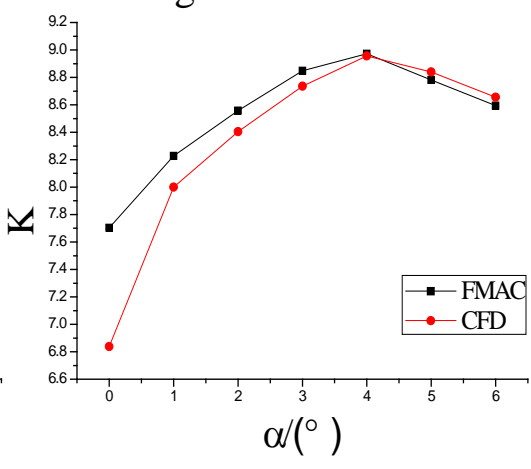

(c) Lift-drag ratio

Figure.7 The Comparison of Aerodynamic Characteristics at Cruise State

With the angle of attack increasing, the lift and drag coefficient maintain increasing. When the angle of attack is about $4^{\circ}$, the lift-drag ratio reaches maximum.

\section{Conclusion}

FMAC for compound gyroplane has been established, and is used for aerodynamic comparison research of a certain compound gyroplane.

The method uses blade-element momentum theory integration method to calculate aerodynamic characteristics of rotor, but engineering estimation method for the fixed wing section. Based on reasonable assumptions, it simplifies the aerodynamic interference between rotor and fixed wing. The calculation error is acceptable for a rapid aerodynamic characteristics evaluation. The method saves a lot of computing time compared with CFD method, and it can be effectively employed for design optimization of compound gyroplane. 


\section{Acknowledgments}

The Fundamental Research Funds for the Central Universities(YWF-15-GJSYS-071) supports this work.

\section{References}

[1] Jay C J. Carter copter aircraft[C]. The 59nd Annual Forum and Technology Display of the AHS. Phoenix, 2003:1-10.

[2]Floros M W, Johnson W. Performance Analysis of the Slowed-Rotor Compound Helicopter Configuration[C]. The AHS 4th Decennial Specialists' Conference on Aeromechanics. Moffety Field, the American Helicopter Society International. 2009, 1: 1-20.

[3]Franklin D H. An Overview of Autogiros and the Mc Donnell XV-1Convertiplane[R]. NASA/CR-2003-212799.

[4] Harris F D. Rotor Performance at High Advance Ratio; theory versus test[R]. NASA/CR-2008 -215370 .

[5] Vu N A, Lee Y, Lee F, et al. Configuration Design and Optimisation Study of a Compound Gyroplane[J]. Aircraft Engineering and Aerospace Technology, 2011, 83(6): 420-428.

[6] McCormick B W. A Numerical Analysis of Autogyro Performance[R]. AIAA-2002-5950.

[7] Kim H Y, Sheen D J, Park S O. Numerical Simulation of Autorotation in Forward Flight[J]. Journal of Aircraft, 2009, 46(5): 1642-1648.

[8] Li C H, Xu G H. The Rotor Free-Wake Analytical Method for Tilt Rotor Aircraft in Hover and Forward Flight[J]. Acta Aerodynamica. Sinica, 2005, 23(2): 152-156(in Chinese).

[9] Cao Y H. Helicopter Flight Mechanics[M]. Beijing: Beihang University Press, 2005: 55-56(in Chinese).

[10] Ma D L, Ma TL, Liu W W. Aerodynamic Prediction and Wind Tunnel Test Study of UAV[J]. Journal of Beijing University of Aeronautics and Astronautics, 2006, 32(12): 1399-1403(in Chinese).

[11]“Aeroplane Design Handbook” General Editor Committee. Aeroplane Design Handbook(6)-Design of Aerodynamics[M]. Beijing: Aeroplane Industry Press, 2002: 190-322(in Chinese).

[12]“Aerodynamics Handbook” General Editor Committee. Aerodynamics Handbook(2)[M]. Beijing: Aeroplane Industry Press, 1995: 401-824(in Chinese).

[13]Lu Z L, et al. Aerodynamics[M]. Beijing: Beihang University Press, 2009: 135-205(in Chinese).

[14] Harendra P B, Joglekar M J, Gaffey T M, et al. A Mathematical Model for Real Time Light Simulation of the Bell Model 301 Tilt Rotor Research Aircraft[R]. NASA/CR-114614.

[15] Leishman J G. Development of the Autogiro: a Technical Perspective[J]. Journal of Aircraft, 2004, 41(4): 765-781. 